\title{
A Reduced-Size Wide Slot Antenna for Enhancing Along-Body Radio Propagation in UWB On-Body Communications
}

\author{
Wen-Tron Shay, Member, IEEE, Shiou-Chiau Jan, and Jenn-Hwan Tarng, Senior Member, IEEE
}

\begin{abstract}
In this paper, a 6-10-GHz ultrawideband (UWB) directional reduced-size wide slot antenna (RWSA) for on-body wireless communications is proposed and investigated. The RWSA is designed to be narrow in width and can be mounted on the side of a wireless device. When a side-mounted RWSA is worn on a body, the antenna boresight is naturally directed along the body surface, and most of the energy can be efficiently transmitted by direct or diffracted waves. Consequently, the on-body channel performance can be effectively improved. After a series of on-body channel measurements, the results show that, with the side-mounted RWSAs, the path loss is significantly improved (greater than $20 \mathrm{~dB}$ ) compared with the results of previous studies, and the sensitivity of the channel to the human body and environment is effectively lessened. An approach to estimate the variation range of path loss in real environments is also proposed at the end of this paper.
\end{abstract}

Index Terms-Body area network (BAN), channel, path loss, ultrawideband (UWB) antenna, wireless communication.

\section{INTRODUCTION}

$\mathbf{U}$ SING wireless devices around the body for medical and nonmedical uses are promising new applications. The large diversity and potential of these applications have made wireless body area network (BAN) an exciting research topic in wireless communications. To satisfy the technical requirements, the IEEE 802.15 Task Group (TG) 6 was formed for BAN in 2007. The TG 6 is devoted to developing a communication standard for low-power devices and applicable to operations on, in, or around the human body. References [1]-[6] report notable achievements of the TG 6. Several frequency bands are proposed for different service scenarios in [1]. According to [1], the scope of this paper is categorized as scenarios S4 and S5, which stand for line-of-sight (LOS) and non-line-of-sight (NLOS) on-body (body surface to body surface) communications, respectively. The corresponding channel model is CM3 with ultrawideband (UWB) frequency band.

Manuscript received January 12, 2013; revised September 30, 2013; accepted November 27, 2013. Date of publication December 12, 2013; date of current version February 27, 2014. This work was supported by the National Science Council of the ROC under Contract NSC 99-2221-E-009-028-MY2.

W.-T. Shay is with the Institute of Communications Engineering, National Chiao-Tung University Hsinchu, 300, Taiwan and also with the Center for Measurement Standards/ITRI, Hsinchu 300, Taiwan (e-mail: wtshay@itri.org.tw).

S.-C. Jan and J.-H. Tarng are with the Institute of Communications Engineering, National Chiao-Tung University, Hsinchu 300, Taiwan (e-mail: shiouchiau@gmail.com; j4t@mail.nctu.edu.tw).

Color versions of one or more of the figures in this paper are available online at http://ieeexplore.ieee.org.

Digital Object Identifier 10.1109/TAP.2013.2294859
In on-body communications, the presence of the body strongly influences various antenna characteristics, primarily the impedance matching and radiation pattern. Particularly, the antenna radiation pattern determines the dominant propagation mode and path, consequently strongly influencing the channel performance (such as path loss and sensitivity to the environment).

Therefore, the antenna used in on-body communications is crucial, and designing an efficient on-body antenna is challenging. The primary purpose of this study is to develop a UWB antenna, which is less sensitive to the human body, can effectively minimize path loss and influences from the environment, and can be easily integrated into wireless devices. Finally, it is expected to improve the on-body channel performance with the developed antennas.

Several types of UWB antennas have been proposed for on-body research in previous studies. In [7], textile antennas operating over the entire UWB were reported. The antennas can be directly integrated into clothing and have excellent transient characteristics. Monopole-like antennas that exhibit omnidirectional patterns are appropriate for use in on-body wireless links when the antennas are positioned perpendicularly to the body surface. However, this type of antenna is too large for practical implementation. In [8], a low-profile inverted truncated annular conical dielectric resonator antenna (DRA) with wide bandwidth and stable monopole-like patterns was proposed. The low-profile feature enables the DRA to be potentially useful in on-body applications. Goto and Iwasaki [9] proposed a finger-ring UWB monopole antenna; this novel, wearable antenna may increase the diversity of wireless applications. Loop antennas can also be applied to BANs. Yazdandoost and Hamaguchi [10] proposed a small loop antenna printed on a silicon substrate; the antenna is small enough to be fit into medical BAN devices. The effects of human body tissues on the loop antenna were also analyzed.

If the feasibility of integrating antennas into modern commercial wireless devices is considered, printed planar antennas are preferred because of their low form factor and ease of fabrication. Several UWB planar antennas have been used in on-body studies [11]-[17]; however, because of their large sizes, the antennas were worn parallel, rather than vertically, to the body during measurements. In this mounting configuration, the body blocks one side of the radiation of any omnidirectional planar antenna. The resultant radiation pattern is directional and the direction of the main beam is oriented outward from the body [16]. For a directional planar antenna, the body may not have strong influence to its patterns [17], but the boresight is still aimed 
outward from the body. Therefore, when any antenna used in [11]-[17] is employed in on-body communications, most energy radiates away from the body. This results in a high path loss because the direct EM energy transmission along the body surface (shortest distance between the antennas) is relatively small. Furthermore, channel performance could be sensitive to the environment because signal transmissions highly rely on the reflections of nearby scatterers. If any of these antennas is mounted on the front of a device, the device would alter the antenna characteristics, similar to the way the body does.

Chahat et al. [18] proposed a compact planar UWB monopole antenna. This small antenna can be placed perpendicularly to the body, which can effectively reduce the influence that the body exerts on the antenna. Consequently, satisfactory on-body propagations were obtained using this antenna. However, if this compact antenna is integrated into a wireless device, its characteristics would be strongly influenced by the device because there is no shield between the antenna and the device.

Compared with the previous studies, one distinct motivation in this paper is that the goal is to develop an antenna that is suitable for integration in commercial products. Directional planar antennas are preferred in this research because they exhibit lower susceptibility to influences caused by their backside objects, compared with omnidirectional planar antennas. Then, the most important issue becomes that of designing a directional planar antenna which is appropriate in size and works efficiently.

In this paper, a directional reduced-size wide slot antenna (RWSA) is proposed; its narrow width allowed it to be mounted on the side of a wireless device. When a side-mounted RWSA is worn on a human body, the antenna is positioned perpendicularly to the body, and the antenna boresight is naturally directed along the body surface, allowing more electromagnetic (EM) energy to be transmitted along a direct path. Consequently, using side-mounted RWSAs can achieve highly efficient on-body communications. Furthermore, a side-mounted RWSA may not increase the device's thickness, and there is an extra advantage. Most modern wireless devices generally have a large front display. A side-mounted antenna is favorable because it requires less space on the device front.

A distinct feature of the proposed RWSA is that a reflector and a folded ground are introduced to enhance the impedance bandwidth. A wide impedance bandwidth $(S 11<-10 \mathrm{~dB})$ over the frequency range of $6-10 \mathrm{GHz}$ is achieved. After a series of on-body channel measurements, the results show that, with the fabricated RWSAs, the channel performance can be substantially improved.

In this paper, Section II presents an introduction of the RWSA design. Section III presents the measured characteristics of a fabricated RWSA. Finally, Section IV presents a description of on-body channel measurements and their results. In this section, a measurement approach is proposed that can estimate the variation range of channel path loss in real environments. This approach provides an effective measure for comparing the performances of antennas.

\section{Antenna Design}

The idea of side-mounted antenna is promising. However, designing a directional planar antenna with a narrow width and simultaneously achieving a predetermined goal of impedance

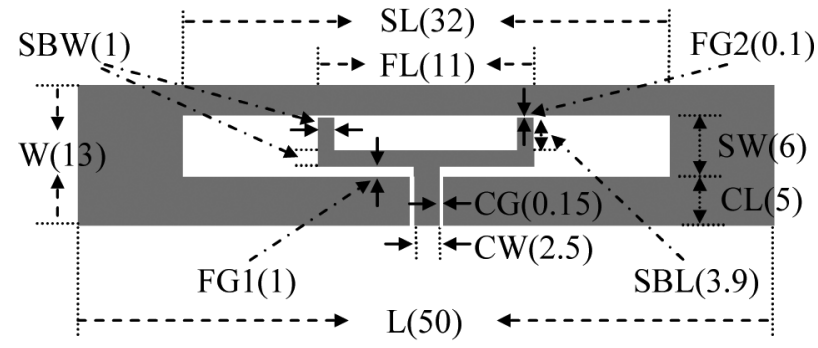

(a)

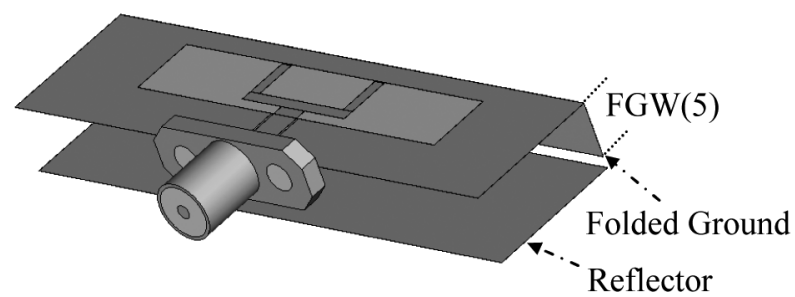

(b)

Fig. 1. Structure of the RWSA and its major dimensions (unit in millimeter): (a) the antenna element; and (b) the entire configuration, comprising the reflector and the extended folded ground (detailed parameter descriptions are listed in Table I).

bandwidth $(6-10 \mathrm{GHz})$ is challenging. Among wideband planar antennas, the concept of wide slot antenna (WSA) was chosen because of the following reasons. First, the impedance bandwidth can be enhanced with a fork-like tuning stub [19], and achieving UWB bandwidth has been reported [20], [21]. Second, the radiation pattern can be modified from omnidirectional to directional by introducing a reflector [22]. Third, the antenna exhibits stable gain patterns and good time-domain responses [23].

Nevertheless, conventional WSAs are too large to be side-mounted. If the antenna width is shortened directly, the impedance matching will be severely deteriorated; solving this problem is the primary challenge in the antenna design.

The structure of the proposed RWSA is shown in Fig. 1. The antenna is printed on a $0.813-\mathrm{mm}$-thick Rogers RO4003 substrate with a dielectric constant of 3.38. The wide slot is fed by a coplanar waveguide (CPW) with a fork-like tuning stub. A CPW feeding structure was chosen because of easier fabrication. As mentioned above, shortening the antenna width directly will cause the loss of wide impedance bandwidth. For an RWSA, a $6-10-\mathrm{GHz}$ bandwidth is not obtainable by simply tuning the dimensional parameters shown in Fig. 1(a). The key in this design is introducing a reflector and an extended folded ground into the antenna, as shown in Fig. 1(b). The original function of the reflector is to block the backward radiation and, therefore, reduce the interaction between the antenna and its backside objects (e.g., the user's body or electronic devices). However, the input impedance of a WSA is strongly influenced by introducing a reflector, especially when the spacing between the antenna element (fork-like tuning stub and ground) and the reflector is small (such as $<10 \mathrm{~mm}$ ). Typically, adding a reflector to a matched WSA degrades its impedance matching [22]. However, in this study, in addition to blocking the backward radiation, the reflector is also used to transform the input impedance of a poorly matched shortened WSA. With appropriate design, the originally poor impedance matching could be significantly 


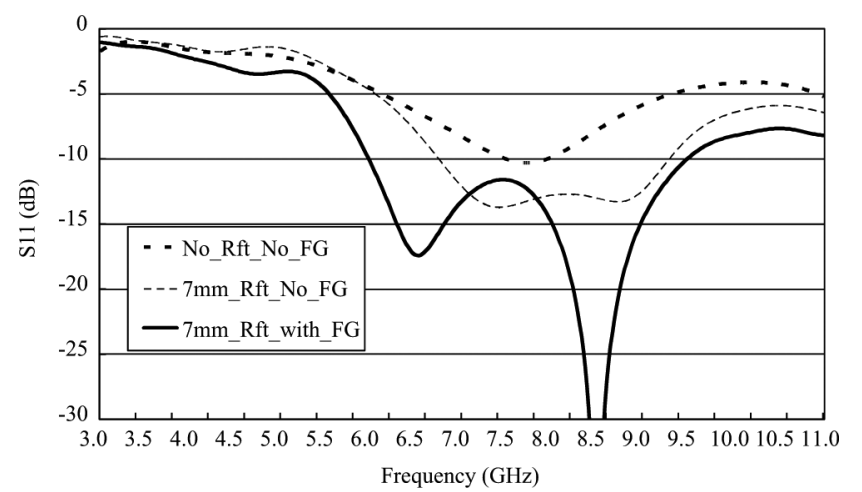

Fig. 2. Impedance bandwidth enhanced by introducing a reflector and a folded ground.

improved. Moreover, a larger ground could also enhance the impedance bandwidth. To avoid enlarging the antenna size, a folded ground is introduced to the antenna. Simulated results (with CST Microwave Studio) demonstrate how the reflector and the folded ground improve the impedance bandwidth, as shown in Fig. 2. Without the reflector and the folded ground, the reflection performance is poor (No_Rft_No_FG). After placing a reflector at a distance of $7 \mathrm{~mm}$, the impedance bandwidth of the antenna is significantly improved (7 mm_Rft_No_FG). If a folded ground is added, the impedance bandwidth is further enhanced ( $7 \mathrm{~mm}$ Rft_with_FG). The bandwidth improvement is due to two resonances introduced by the reflector and the folded ground.

The antenna element is printed on the side facing the reflector so that the substrate can prevent the antenna element from being touched by objects, such as fingers. In Fig. 1(b), the substrate is set to be transparent to visualize the antenna element.

In addition to introducing the reflector and the folded ground, more elaborate design was required to achieve a wide impedance bandwidth. During the design process, the antenna length (L) was fixed, and the antenna width (W) and the slot width (SW) were gradually reduced. After the predetermined 6-10-GHz impedance bandwidth was achieved at a selected width (W or SW), the width was then reduced by $1 \mathrm{~mm}$, and the next design process was carried on. This procedure explains why a predetermined impedance bandwidth is required. All of the dimensional parameters shown in Table I have influences on the impedance matching. Among the parameters, the slot length (SL), the fork-like stub length (FL), the spacing (SP), the folded ground width (FGW), and the stub branch section length (SBL) are critical; thus, these parameters are discussed in the following paragraphs. The remaining parameters were determined after several preliminary simulations and kept invariant throughout the design process.

In the following analyses, the dimensional parameters were properly selected to obtain two resonance frequencies. Subsequently, the value of one selected parameter was varied to investigate its influence on the reflection coefficients ( $S 11$ in decibels).

Fig. 3(a) and (b) shows the effects of varying the SL and FL, respectively. It is observed that the upper resonance frequency of the antenna shifts higher as the SL or FL is reduced. The SL and FL show similar effects on the reflection coefficients. These
TABLE I

DimenSIONAL PARAMETERS OF THE RWSA

\begin{tabular}{lcc}
\hline \hline \multicolumn{1}{c}{ Antenna Dimensional Parameters } & $\begin{array}{c}\text { Initial } \\
\text { Value (mm) }\end{array}$ & $\begin{array}{c}\text { Final } \\
\text { Value (mm) }\end{array}$ \\
\hline Antenna length (L) & 50 & 50 \\
Antenna width (W) & 50 & 13 \\
Antenna thickness (T) & 9 & 7 \\
Substrate thickness (ST) & 0.813 & 0.813 \\
Slot length (SL) & 32 & 32 \\
Slot width (SW) & 21 & 6 \\
Reflector length (equal to L) & 50 & 50 \\
Reflector width (equal to W) & 50 & 13 \\
Feeding CPW length (CL) & 15 & 5 \\
CPW center conductor width (CW) & 2.5 & 2.5 \\
CPW slot width (CG) & 0.15 & 0.15 \\
Gap width, between horizontal section and & 1 & 1 \\
ground (FG1) & & \\
Gap width, between branch sections and & 10 & 0.1 \\
ground (FG2) & & 11 \\
Fork-like stub horizontal section length & 16 & 3.9 \\
(FL) & 10 & 1 \\
Fork-like stub branch section length (SBL) & 2 & 50 \\
Fork-like stub line width (SBW) & none & 5 \\
Folded ground length (equal to L) & none & \\
Folded ground width (FGW) & 8 & \\
Spacing between antenna element and & & \\
reflector (SP) & & \\
\hline \hline
\end{tabular}

results are reasonable because the radiation of the antenna is mainly controlled by the fork-like stub and the slot. As these elements are smaller, it is predictable that the resonance frequency will be higher. The lower resonance frequency is not sensitive to the SL or FL. Therefore, shortening the SL or FL might cause the two resonance frequencies to be separated excessively, and that could result in higher S11 at the frequencies between the two resonance frequencies, for example, frequencies near 7.5 $\mathrm{GHz}$ in Fig. 3(a). In addition, operating frequencies higher than 10.6 GHz are not desired because using these frequencies is not allowed without a license. Therefore, the SL and FL cannot be too short.

Fig. 3(c) shows the effect of varying the SP. It is found that reducing the SP also shifts the upper resonance frequency higher. Smaller SP is preferred because the thickness of the antenna can be reduced. However, when the reflector is moved closer to the antenna element, the impedance bandwidth becomes narrower. This might be because the increased capacitance leads to more energy stored in the antenna, which increases the quality factor $Q$ of the antenna. By contrast, increasing the SP reduces the effect of the reflector and the impedance matching becomes poorer, as shown in Fig. 3(c) $(\mathrm{SP}=14 \mathrm{~mm})$.

Fig. 3(d) shows the effect of varying the FGW. As the FGW increases, the lower resonance frequency shifts lower. This implies that larger ground could allow resonance at lower frequencies. It is also observed that the upper resonance frequency is not sensitive to the FGW, which is different from the effects by varying the SL, FL, or SP. Increasing the FGW also causes larger separation between the two resonance frequencies, and the matching at the frequencies between the two resonance frequencies becomes worse. The folded ground must not contact the reflector; the condition FGW $<$ SP must be met. This could be solved with a multifolded ground, at the cost of increasing the complexity of the antenna. 


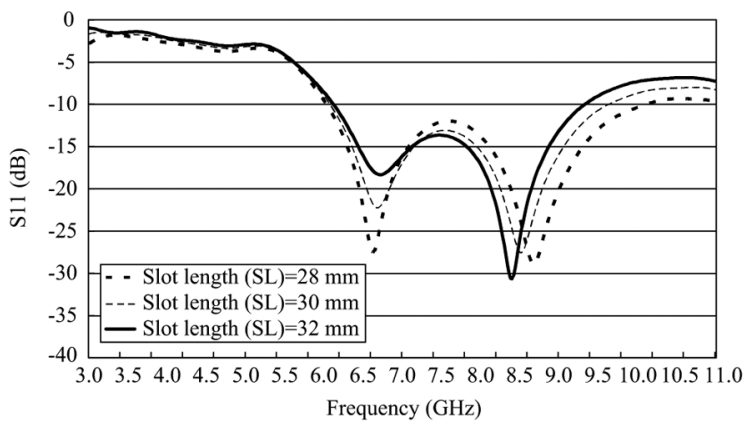

(a)

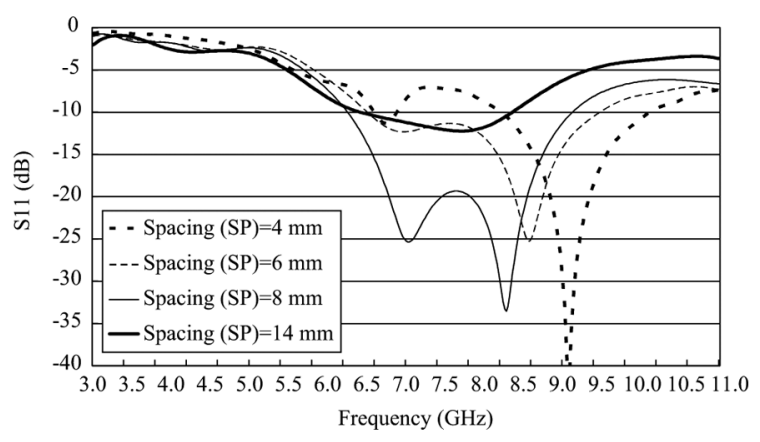

(c)

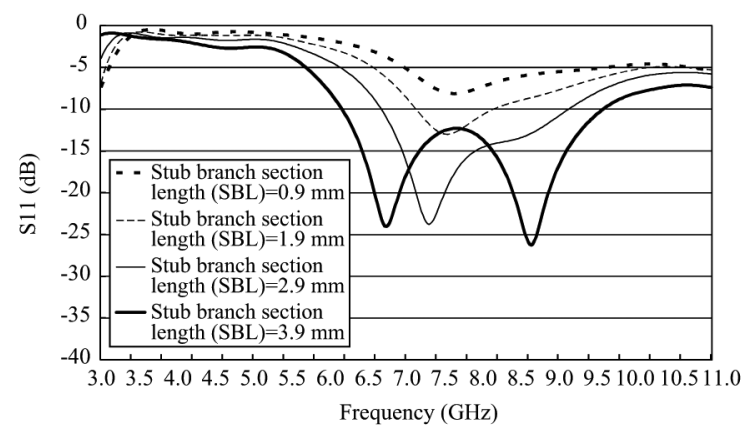

(e)

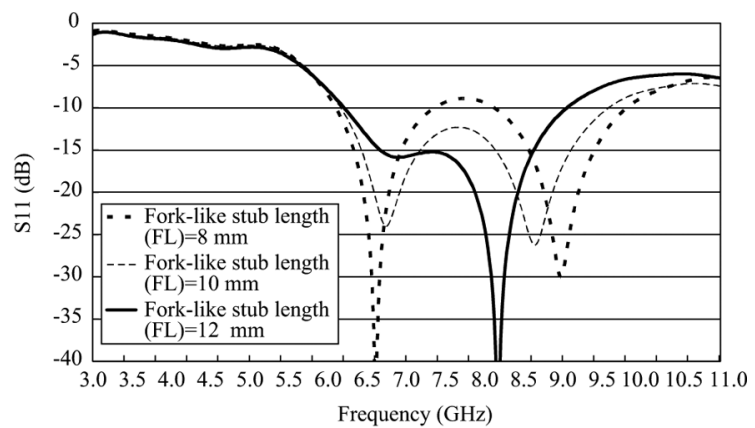

(b)

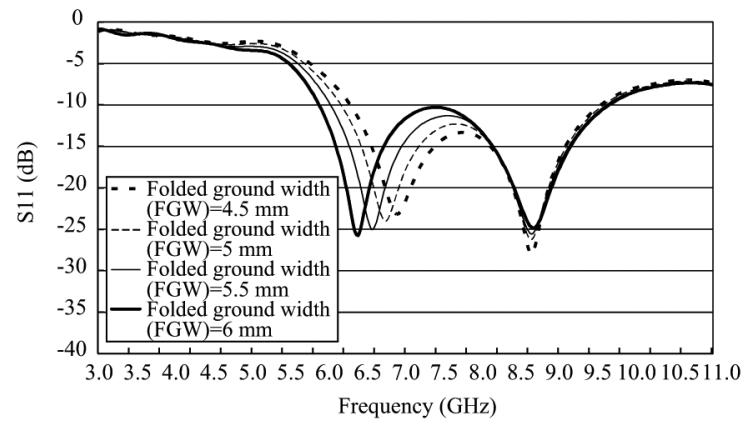

(d)

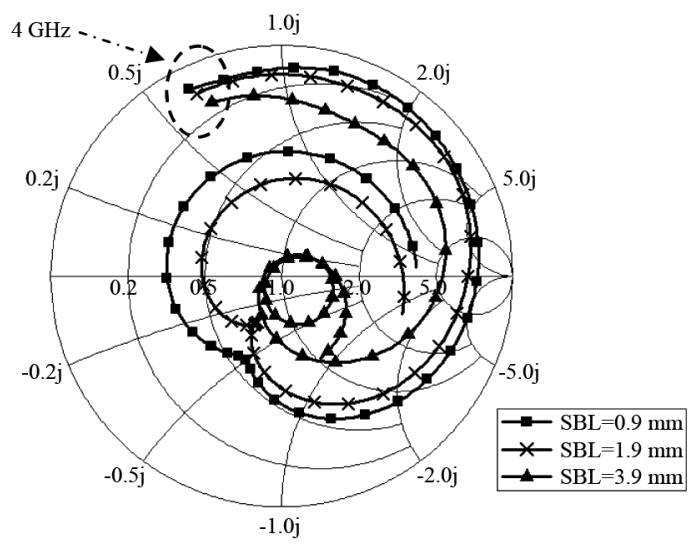

(f)

Fig. 3. Effects of selected dimensional parameters on the reflection coefficient or the input impedance: (a) SL, (b) FL, (c) SP, (d) FGW, (e) SBL, and (f) SBL with a Smith chart.

Fig. 3(e) shows the effect of varying the SBL. It is obvious that the impedance bandwidth is gradually enhanced as the SBL increases. However, the SBL is limited by the slot width because the branch sections must not contact the ground. The character and the function of a branch section are similar to those of a shunt open stub in a microwave circuit. It could provide reactance for impedance matching. For an open stub, the first maximum reactance occurs when the length of the stub is equal to $1 / 4$ wavelength $(\lambda)$ of the operating frequency, and the reactance gradually reduces as the stub length reduces. If an antenna is initially far from matching, introducing a large reactance might be necessary to transform the input impedance. Fig. 3(f) shows the effect of varying the SBL with a Smith chart (from 4 to $10 \mathrm{GHz}$ ). It can be seen that increasing the SBL can effectively transform the input impedance closer to the center (matching) at higher frequencies. For lower frequencies, the SBL is relatively short if compared with the wavelength; thus, the effect of the branch sections is not significant. In this design, the final selected SBL is $3.9 \mathrm{~mm}$, which is near, but shorter than $\lambda / 4$ of $10 \mathrm{GHz}$. Shortening the antenna width will also shorten the SBL. Consequently, the reactance introduced by the shortened branch sections might be too small to improve the impedance matching effectively. Therefore, the SBL might limit the minimum width of the RWSA.

The above analyses were conducted to investigate the influences of some selected parameters by varying only one selected parameter at a time while holding the others fixed. The results provide the basic guidelines in the antenna design. However, modifying one parameter alters the effects of other parameters on the input impedance. Therefore, the design was cumbersome. After extensive simulations and repeated tuning in measurements, the final optimized dimensions were obtained. Table I lists the initial and final dimensions of the RWSA. The final size of the RWSA is $50(\mathrm{~L}) \times 13(\mathrm{~W}) \times 7(\mathrm{~T})$ in millimeters, which 


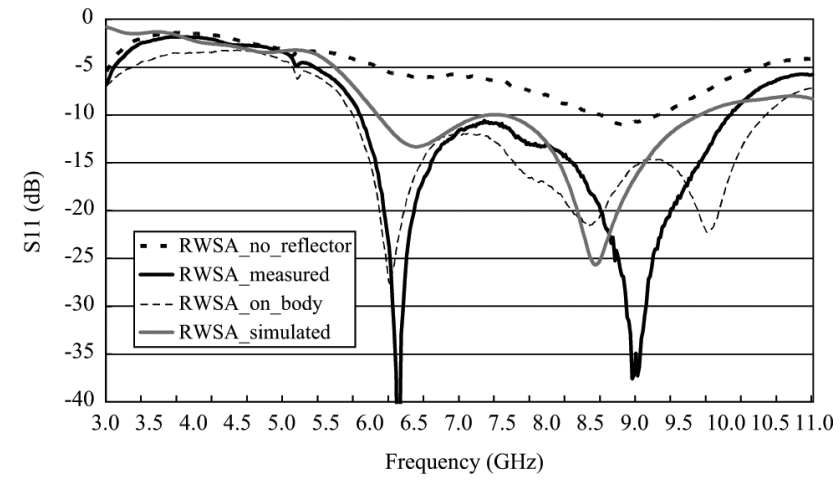

Fig. 4. Measured and simulated reflection coefficients of an RWSA.

is acceptable for general wireless devices. The feasibility of reducing the antenna dimensions is analyzed as follows. The antenna length $(\mathrm{L})$ is not critical and it can be slightly reduced. The antenna thickness $(\mathrm{T})$ is mainly determined by the spacing (SP), and the SP is related to the impedance bandwidth. Reducing the SP also alters the usable frequencies. Minimizing the antenna width (W) to a favorable size and still achieving the predetermined impedance bandwidth is the main effort of the antenna design. The value $13 \mathrm{~mm}$ includes the length of the CPW that is used to connect an SMA connector. Thus, the antenna width could be further shortened when an RWSA is integrated in a commercial device because no connector is required for this condition. With modern circuit connection techniques, such as flexible printed circuits, an antenna width shorter than $10 \mathrm{~mm}$ could be achievable.

\section{ANTENNA ChARACTERISTICS}

\section{A. Reflection Coefficient}

The measured and simulated reflection coefficients of one fabricated RWSA are shown in Fig. 4. The measured results (in free space) show that the frequency band for $S 11<-10 \mathrm{~dB}$ is from 5.9 to $10.1 \mathrm{GHz}$ (RWSA_measured), which covers the entire design goal $(6-10 \mathrm{GHz})$. The corresponding impedance bandwidth is approximately $52 \%$. When the RWSA is placed on a body, the resonance frequencies are slightly shifted (RWSA_on_body), but the impedance bandwidth is maintained (slightly wider than in free space). These measured results show that the impedance matching of the RWSA is only slightly influenced by the body. This is expected because the antenna element is perpendicular to the body surface when the RWSA is side-mounted. The importance of the reflector is also illustrated by the measured results shown in Fig. 4. Without a reflector, the measured impedance bandwidth of the RWSA is quite narrow (RWSA_no_reflector). Fig. 4 also shows that the measured and simulated reflection coefficients of the RWSA are matched. The measured impedance bandwidth is slightly wider. This might be because of the conductor loss which was not considered in the simulation.

\section{B. Radiation Pattern}

The measured radiation patterns of a fabricated RWSA at 6, 7, 8, 9, and $10 \mathrm{GHz}$ are shown in Fig. 5. The antenna exhibits directional behavior across the entire frequency band, and its peak gains are high, as shown in Table II. The averaged 3-dB

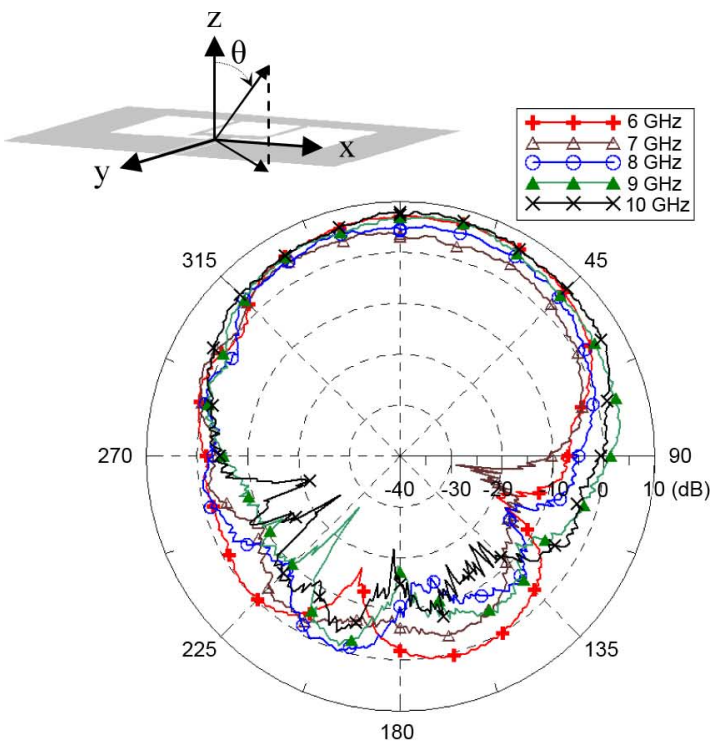

(a)

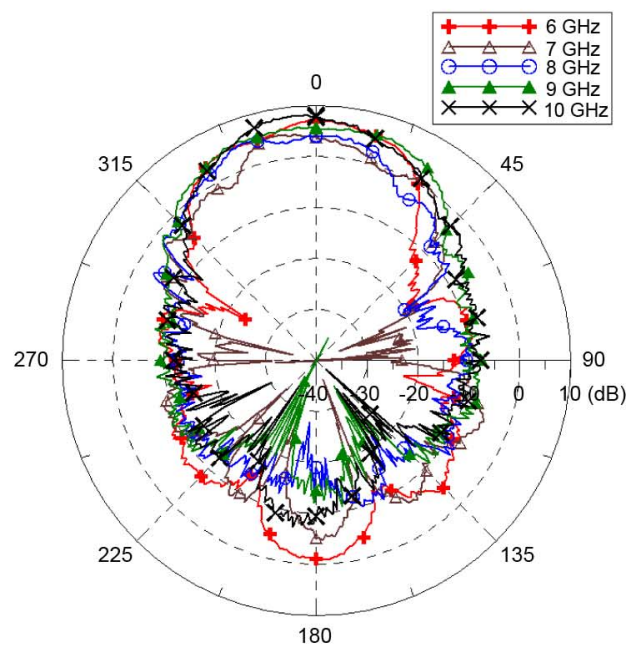

(b)

Fig. 5. Radiation patterns of an RWSA at 6, 7, 8, 9, and $10 \mathrm{GHz}$ : (a) the E-plane $(y z)$ pattern, and (b) the H-plane $(x z)$ pattern.

TABLE II

PEAK GaINS OF THE RWSA

\begin{tabular}{|c|c|c|c|c|c|}
\hline Frequency $(\mathrm{GHz})$ & 6 & 7 & 8 & 9 & 10 \\
\hline Measured Peak Gain $(\mathrm{dBi})$ & 7.3 & 4.7 & 5.6 & 7.4 & 8.1 \\
\hline
\end{tabular}

beamwidth of the E-plane $(y z)$ and H-plane $(x z)$ are approximately $104^{\circ}$ and $44^{\circ}$, respectively. It is observed that the patterns at different frequencies are similar. This is important because it implies that if RWSAs are used, the received UWB pulse would suffer less distortion caused by the variations in antenna patterns.

To investigate the influence of a human body on the antenna, the radiation patterns were measured as the RWSA was placed on a rectangular plastic container filled with saline $(9 \mathrm{~g} \mathrm{NaCl}$ per liter). The measured dielectric constant and loss factor of the saline at $8 \mathrm{GHz}$ are 65 and 29, respectively. Fig. 6 presents the measured radiation patterns at 6,8 , and $10 \mathrm{GHz}$. Because of the limitations of the antenna test system, a saline-filled container was used rather than a human body. However, the shape 


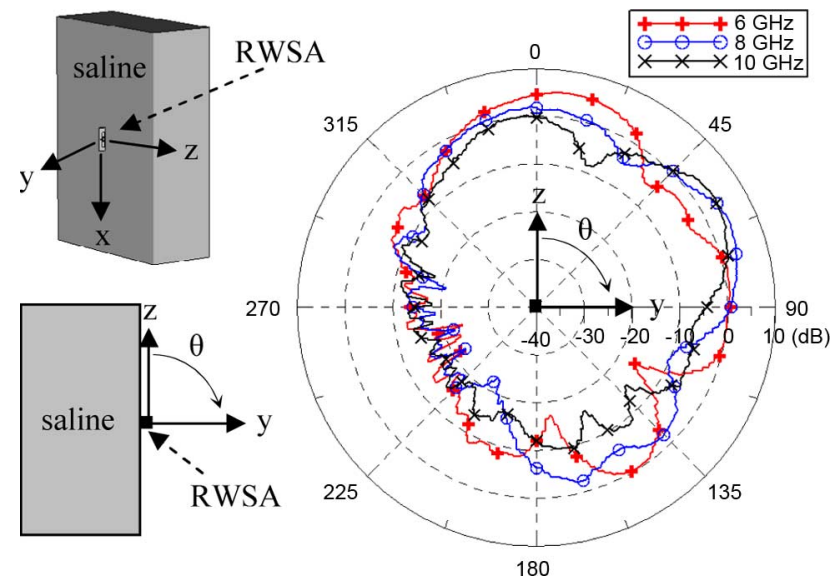

Fig. 6. Schematic view of an RWSA attached to a container (upper left), the top view (lower left), and the E-plane radiation patterns at 6,8 , and $10 \mathrm{GHz}$ (right).

of the container is invariant and the antenna can be precisely placed. Therefore, a better measurement repeatability and reliable comparison between antennas can be obtained. In the $z$-direction (along the container surface), the gains for all frequencies are not less than $0 \mathrm{dBi}$. In addition, the gains in the directions blocked by the container are still favorable because of diffraction effect. For example, the gains in the $345^{\circ}-360^{\circ}$ directions are higher than $-1.5 \mathrm{dBi}$. These results imply that the antenna could still effectively transmit energy along and around the torso, when the antenna is worn on a body.

\section{ON-Body Measurement AND Result}

\section{A. Path Loss Measurement}

In this study, a vector network analyzer (VNA) was used to measure the $S 21$ parameter between two antennas worn on a test person. The test person was asked to keep standing still during the measurements. The measurement frequency range was from 6 to $10 \mathrm{GHz}$. For achieving better measurement repeatability, each RWSA and its connecting cable were secured with a plastic fixture, as shown in Fig. 7. As mentioned previously, the antenna element is printed on the inner side of the substrate and, therefore, cannot be seen in Fig. 7. Around and along the torso measurements were performed in this study. The antenna positions are depicted in Fig. 8. Regarding the measurements around the torso, measurements were performed at six planes separated by $5 \mathrm{~cm}$ along the vertical direction of the torso. For each plane, the transmitting antenna (circle) was placed on the right-hand side of the waist, and the receiving antenna (square) was placed in various positions around the torso. The first position was 10 $\mathrm{cm}$ away from the transmitting antenna and the distance increased in an increment of $5 \mathrm{~cm}$ for the rest of the positions. The maximum distance between the two antennas was $45 \mathrm{~cm}$; longer than this distance, the receiving antenna approached the transmitting antenna from the other side. For the measurements along the torso, the transmitting antenna was placed near the shoulder in six positions separated by $5 \mathrm{~cm}$. When the transmitting antenna was in one position, the receiving antenna was placed directly below it in various positions. The distance of the first position was also $10 \mathrm{~cm}$ and the distance increased in

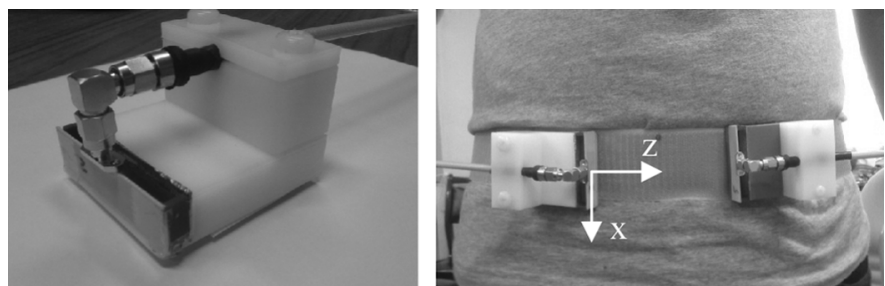

Fig. 7. Configured RWSA (left), and positions of the antennas for the around the torso measurements (right).

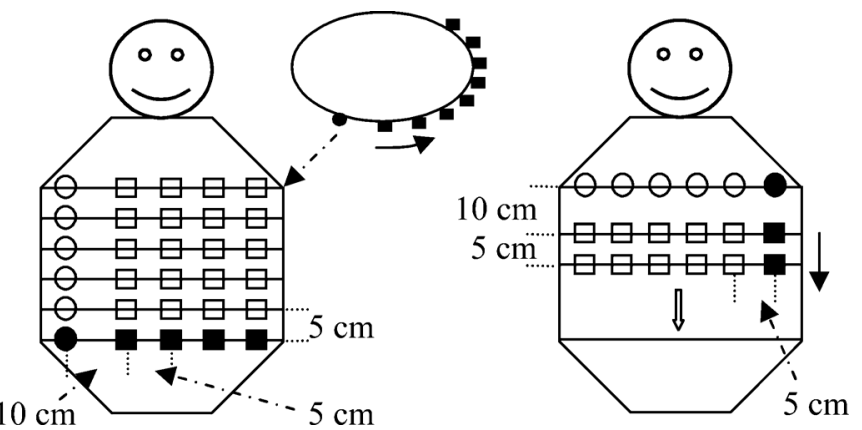

Fig. 8. Positions of the antennas for around the torso (left) and for along the torso (right) measurements.

an increment of $5 \mathrm{~cm}$ for the rest positions. Twenty five measurements were taken for each position of the receiving antenna (for both the around and along the torso scenarios). To reduce the effect of small body movements during measurements, the averaged value was taken as the path loss in that position. The averaging process can also reduce the influences of the cables. The path loss variation caused by the long cables was evaluated by repeatedly measuring the loss of the two directly connected cables. During the measurements, the cables were placed in various positions to simulate the conditions in real on-body measurements [24]. Based on 1000 measured data, the maximum variation of the loss was $0.5 \mathrm{~dB}$ when no averaging was applied and $0.1 \mathrm{~dB}$ when the averaging was applied.

To investigate the influence of separation between the antenna and the body on the path loss, the separation was varied by either placing the RWSAs directly on the body (spaced only by a Velcro strip and clothes), or adding a 5-mm (thickness) low dielectric constant plastic between the RWSAs and the body. The measurement arrangements were similar to [11] for convenience of the following comparisons.

The path loss at a distance $d$ (from the transmitting antenna) is modeled with [1]

$$
\mathrm{PL}_{\mathrm{dB}}(d)=\mathrm{PL}_{0, \mathrm{~dB}}+10 n \log \left(\frac{d}{d_{0}}\right)
$$

where $n$ is the path loss exponent, $d_{0}$ is the reference distance, and $\mathrm{PL}_{0, \mathrm{~dB}}$ is the path loss at the reference distance. The measured path loss is the power loss between the transmitting antenna connector and the receiving antenna connector. Although it is not explicitly expressed in (1), the on-body path loss parameters, derived from the measured results, include the effects of the transmitting and receiving antennas. Therefore, it is a convenient method to evaluate an antenna performance by examining the path loss parameters.

Fig. 9(a) shows the results of the around and along the torso measurements in an office. The path loss differences between 


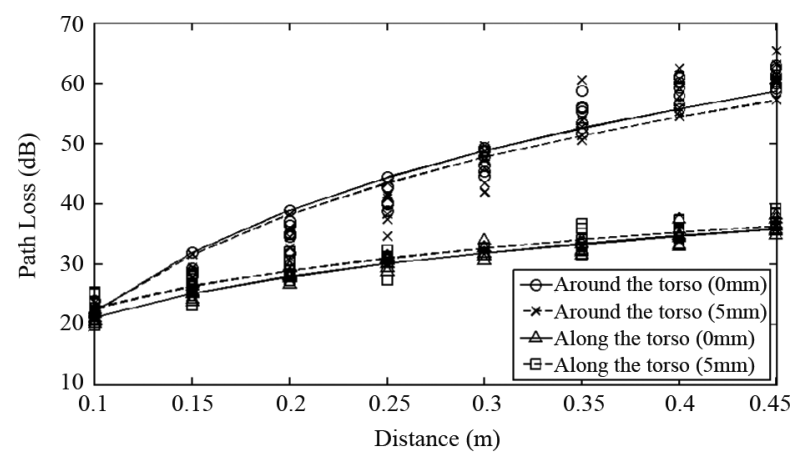

(a)

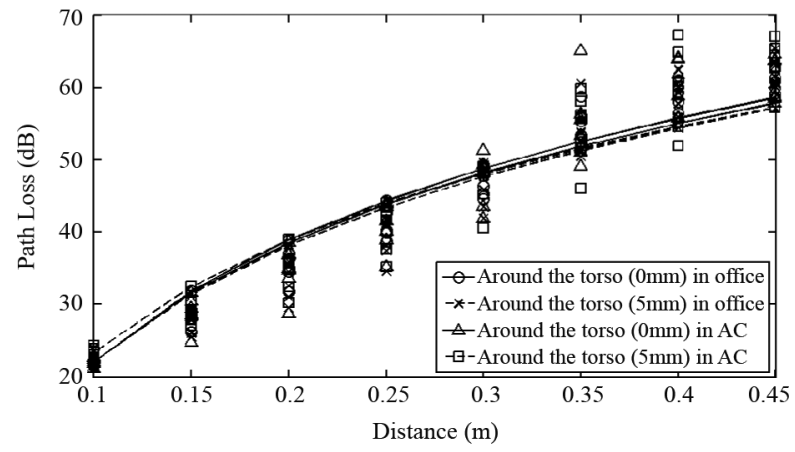

(b)

Fig. 9. (a) Measured path loss for around and along the torso scenarios in an office, with and without a 5-mm plastic. (b) Measured path loss for around the torso scenario in an office and in an anechoic chamber.

the results, with $(5 \mathrm{~mm})$ and without $(0 \mathrm{~mm})$ the plastic, are rather small for both measurement scenarios. These results indicate that the separation has no obvious influence on the path loss. For the around the torso measurement, there is an abrupt increase (breakpoint) of path loss between 0.3 and $0.35 \mathrm{~m}$. This is because the radio propagation is changing from LOS to NLOS. In fitting the path loss curve, LOS and NLOS data were not separated. The reason is because it is unlikely to distinguish them in practical applications. Therefore, the fitted curve may not pass the clusters of points at certain distances. It is obvious that the path loss around the torso is higher than that along the torso. This result is reasonable because the radio propagation is always in LOS condition for the along the torso scenario. To investigate the influence of the environment on the path loss while using RWSAs, around the torso measurements were repeated in an anechoic chamber (AC). The results are compared with those obtained in the office, as shown in Fig. 9(b). The differences are rather small. This outcome is because the energy transmission between the RWSAs mainly relies on direct and diffracted waves. Reflections are less significant. In addition, no large scatterers were near the antennas during the in-office measurements. As can be seen in Fig. 9(a) and (b), it is clear that the channel with the RWSAs is not sensitive to the human body and environment.

The path loss parameters extracted from the measurement results are shown in Table III. The parameters in [1], [11], and [12] are also listed as references. Comparing the parameters (using RWSAs) with the references, the $\mathrm{PL}_{0, \mathrm{~dB}}$ values are improved approximately $25-34$ and $22-35 \mathrm{~dB}$ for around and along the
TABLE III

PATH LOSS MODEL PARAMETERS

\begin{tabular}{|c|c|c|c|c|c|c|}
\hline \multicolumn{7}{|c|}{ Around the torso } \\
\hline \multirow{3}{*}{ Parameters } & \multicolumn{2}{|c|}{$\begin{array}{c}\text { With RWSAs in } \\
\text { AC }\end{array}$} & \multicolumn{2}{c|}{$\begin{array}{c}\text { With RWSAs in } \\
\text { office }\end{array}$} & \multicolumn{2}{c|}{$\begin{array}{c}\text { Reference [1] } \\
{[12]}\end{array}$} \\
\cline { 2 - 7 } & $0 \mathrm{~mm}$ & $5 \mathrm{~mm}$ & $0 \mathrm{~mm}$ & $5 \mathrm{~mm}$ & $0 \mathrm{~mm}$ & $5 \mathrm{~mm}$ \\
\hline$P L_{0, d B}(\mathrm{~dB})$ & 22.1 & 23.4 & 22.1 & 22.1 & 56.1 & 48.4 \\
\hline$d_{0}(\mathrm{~m})$ & 0.1 & 0.1 & 0.1 & 0.1 & 0.1 & 0.1 \\
\hline$n$ & 5.5 & 5.2 & 5.6 & 5.4 & 5.8 & 5.9 \\
\hline
\end{tabular}

\begin{tabular}{|c|c|c|c|c|}
\hline \multicolumn{5}{|c|}{ Along the torso } \\
\hline \multirow{2}{*}{ Parameters } & With RWSAs in office & \multicolumn{2}{c|}{ Reference [1] [11] } \\
\cline { 2 - 5 } & $0 \mathrm{~mm}$ & $5 \mathrm{~mm}$ & $0 \mathrm{~mm}$ & $5 \mathrm{~mm}$ \\
\hline$P L_{0, d B}(\mathrm{~dB})$ & 21.2 & 22.6 & 56.5 & 44.6 \\
\hline$d_{0}(\mathrm{~m})$ & 0.1 & 0.1 & 0.1 & 0.1 \\
\hline$n$ & 2.2 & 2.1 & 3.1 & 3.1 \\
\hline
\end{tabular}

torso scenarios, respectively. The path loss exponents are also reduced by using the RWSAs.

In this study, the results of [11] and [12] were adopted for comparison because the IEEE document [1] cites their results, and their proposed antenna positions are appropriate for studying the LOS and NLOS on-body propagations. Although the frequency range studied in [11] and [12] is 3-6 $\mathrm{GHz}$, the above comparisons are still meaningful because both measurements were under similar arrangements, such as antenna positions on the body and environmental conditions (in offices). At present, parties may freely choose frequency bands (in UWB) for their applications; therefore, comparisons between different frequency bands are both possible and feasible. The comparisons may be useful for system designers to decide the best frequency band for their products.

Table III shows that $\mathrm{PL}_{0, \mathrm{~dB}}(5 \mathrm{~mm})$ might be slightly higher than $\mathrm{PL}_{0, \mathrm{~dB}}(0 \mathrm{~mm})$. The difference is not significant. In our experience, this phenomenon is because of the inherent variations in on-body measurements. The results do not indicate that the path loss performance of the channel with the $5-\mathrm{mm}$ plastic is worse than that without the plastic.

\section{B. Path Loss Range Estimation}

It is found that the scatterers in the vicinity of the antennas have significantly stronger influences on the on-body channels. On-body channel measurements are often performed in indoor environments (such as offices). However, the types and positions of nearby scatterers vary from place to place so that the influences from the surroundings would not be identical. Therefore, comparisons of antenna performances evaluated in different environments may not be sufficiently accurate. To solve this problem, performing path loss measurements both in an anechoic chamber and in a shielding room are proposed. Anechoic chambers and shielding rooms have similar reflection characteristics among rooms of the same type. The two room types represent two extreme conditions. One provides a minimum reflection environment and the other provides a maximum reflection environment. With the measurement results obtained in the two rooms, the variation range of path loss in real environments can be estimated. Therefore, instead of performing numerous measurements in indoor and outdoor environments, the performance of an antenna can be easily 

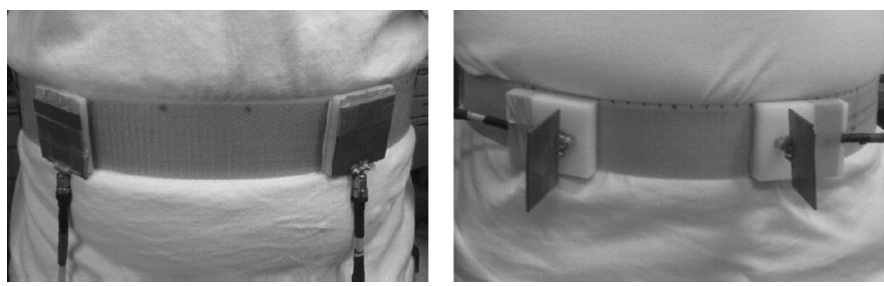

Fig. 10. PICAs parallel (left) and vertical to the test body during measurements.

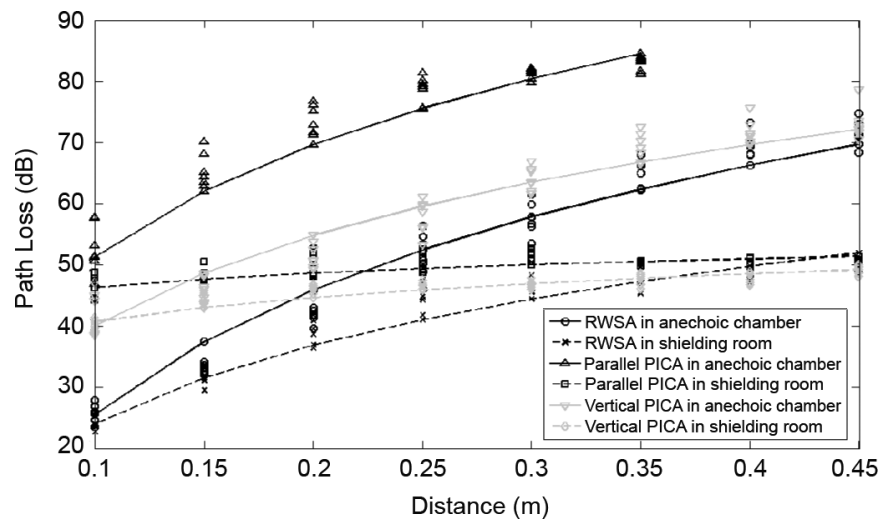

Fig. 11. Comparison of the path loss measured in an anechoic chamber and in a shielding room with RWSAs and PICAs.

evaluated. Using this approach, antennas developed by different parties could be fairly compared by examining the results measured at their own test facilities. This approach might also have practical uses in system designs. For example, people who wear on-body wireless devices may be in an open area or in an elevator. The system designer generally needs to know the variation range of the received power level. By using this method, design parameters can be easily obtained.

Around the torso measurements were carried out in an anechoic chamber and in a $3 \mathrm{~m} \times 1.7 \mathrm{~m} \times 2.4 \mathrm{~m}$ (height) empty shielding room with side-mounted RWSAs (without plastic). In addition, for comparison, two $50 \mathrm{~mm} \times 44 \mathrm{~mm}$ planar inverted cone antennas (PICAs) [15] were also used in the measurements. Typically, PICAs are placed parallel to the body in measurement; however, additional measurements in a vertical configuration were also performed to observe the differences (Fig. 10). Fig. 11 shows the measured results. The differences between using RWSAs and PICAs are clearly seen. When the distance between the RWSAs is short, the direct transmission dominates; and, therefore, the differences of the path loss in the anechoic chamber and shielding room are small. As the distance increases, the reflected waves become significant, and the differences become larger. In comparison with the results shown in Fig. 9(b), it is obvious that the effect of reflections can be observed in the shielding room but cannot in the office. These results explain why a shielding room must be used in antenna performance evaluation. Fig. 11 also shows that the differences of the path loss with the parallel-to-body PICAs (with $5 \mathrm{~mm}$ of plastic) obtained in the two rooms are significantly larger than those with the RWSAs. This is because the transmission between the parallel-to-body PICAs heavily relies on reflected waves. Therefore, environments have stronger influences on the channel. For using the PICAs in both parallel and vertical configurations, the path loss only slightly increases as

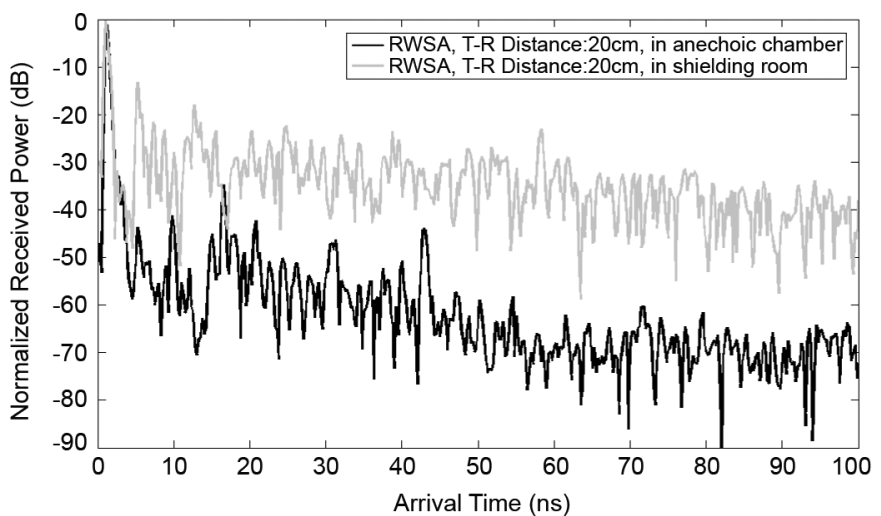

(a)

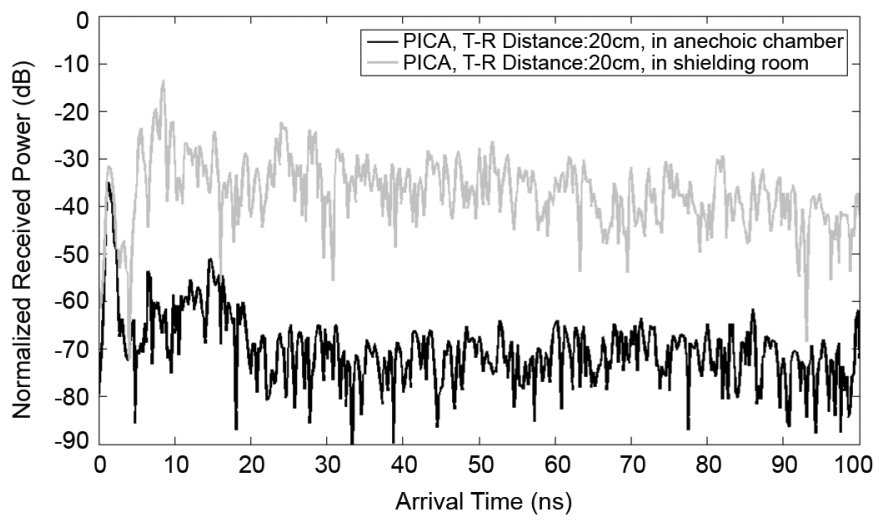

(b)

Fig. 12. Power delay profiles measured in an anechoic chamber and a shielding room, using (a) RWSAs and (b) PICAs.

the distance increases in the shielding room. This phenomenon may be because the path loss primarily depends on the traveled distances of the reflected waves, rather than the antenna separation. Using the vertical-to-body PICAs, the path loss is lower than that using the parallel-to-body PICAs, but higher than that using the RWSAs. It is interesting to find that the measured results of the path loss at $45 \mathrm{~cm}$ are close for the three measurements in the shielding room; this may imply that which antenna is used is not critical in a highly reflective environment, if NLOS signals dominate. The maximum distance for using the PICAs in an anechoic chamber is $35 \mathrm{~cm}$ because the received power level is near the noise floor of the VNA at a distance of $40 \mathrm{~cm}$.

The differences between the two sets of path loss parameters obtained in the two rooms can be used to evaluate the sensitivities (to the environment) of channels, when employing various antennas. For example, the differences $\left(\Delta \mathrm{PL}_{0, \mathrm{~dB}}, \Delta n\right)$ with the RWSAs and with the parallel-to-body PICAs are $(1.5,2.5)$ and $(5,5.6)$, respectively. With these quantitative parameters, it is easy to select a better antenna to obtain a more stable channel for practical applications.

In addition to the path loss, the time-domain characteristics of wave propagation were also studied using RWSAs and PICAs (parallel to body). Fig. 12 shows the power delay profiles measured in the anechoic chamber and shielding room. The separation of the transmitting and receiving antenna was fixed at $20 \mathrm{~cm}$ during the measurements. In Fig. 12, the vertical axis shows the received power normalized to the power level of the strongest received signal when the RWSAs were used in 
the shielding room. As expected, the power level of the first signal received using the RWSAs is substantially higher than that received using the PICAs in either environment. In the shielding room, the power levels of the multipath components are markedly higher than those in the anechoic chamber. A threshold is set at $10 \mathrm{~dB}$ below the strongest arriving signal. For using the RWSAs in both environments and using the PICAs in the anechoic chamber, no multipath components with power levels higher than the threshold are observed. By contrast, for using the PICAs in the shielding room, the power levels of certain multipath components exceed the first received power level. The maximum excess delay, or the time delay during which the multipath power level falls to $10 \mathrm{~dB}$ below the maximum, is 24 ns. Therefore, although the path loss is low when the PICAs are used in the shielding room (Fig. 11), the multipath components may degrade the quality of on-body communications. In summary, the time-domain measurement results indicate that when the RWSAs are employed, the power level of the first received signal is markedly higher than those of the multipath components; therefore, the multipath effect can be mitigated.

\section{CONCLUSION}

In this paper, a $6-10-\mathrm{GHz}$ directional RWSA is proposed and investigated. The RWSA is narrow in width and can be mounted on the side of a wireless device. Using the side-mounted RWSAs allows more direct energy transmission between antennas. After a series of on-body channel measurements, the results show that, with the side-mounted RWSAs, the path loss is significantly improved, and the channel is less sensitive to the human body and environment.

Path loss is crucial for most wireless communications. Lower path loss permits higher transmission capacity, better communication quality, and lower power consumption. The RWSA and the side-mounted antenna concept are proved effective in reducing the path loss. It is expected that the results of this study can benefit the development of on-body communications.

The RWSA is a directional antenna. One RWSA cannot cover every direction along the body. However, this problem can be solved with modern multiple-input-multiple-output (MIMO) technology. It is easy to mount a second RWSA on a different side of the device. In fact, the RWSA is also designed for on-body MIMO studies. The on-body communications are expected to gain benefits from the MIMO technology using RWSAs.

\section{REFERENCES}

[1] Channel Model for Body Area Network (BAN), P802.15-08-0780-110006, IEEE, Sep. 2010, K. Y. Yazdandoost and K. Sayrafian-Pour.

[2] TG6 Technical Requirements Document (TRD), P802.15-08-0644-090006, IEEE, Nov. 2008, B. Zhen, M. Patel, S. Lee, E. Won, and A. Astrin.

[3] IEEE Standard for Local and Metropolitan Area Networks-Part 15.6: Wireless Body Area Networks, IEEE Standard 802.15.6, 2012.

[4] A. Reichman, "Standardization of body area networks," in Proc. IEEE Int. Conf. Microw. Commun. Antennas Electron. Syst., 2009, DOI: 10.1109/COMCAS.2009.5385994.

[5] C. Lee, J. Kim, H. S. Lee, and J. Kim, "Physical layer designs for WBAN systems in IEEE 802.15.6 proposals," in Proc. 9th Int. Symp. Commun. Inf. Technol., 2009, pp. 841-844.
[6] K. S. Kwak, S. Ullah, and N. Ullah, "An overview of IEEE 802.15.6 standard," in Proc. 3rd Int. Symp. Appl. Sci. Biomed. Commun. Technol., Nov. 2010, DOI: 10.1109/ISABEL.2010.5702867.

[7] M. Klemm and G. Troester, "Textile UWB antennas for wireless body area networks," IEEE Trans. Antennas Propag., vol. 54, no. 11, pt. 1, pp. 3192-3197, Nov. 2006.

[8] G. Almpanis, C. Fumeaux, J. Fröhlich, and R. Vahldieck, "A truncated conical dielectric resonator antenna for body-area network applications," IEEE Antennas Wireless Propag. Lett., vol. 8, pp. 279-282, 2009.

[9] H. Goto and H. Iwasaki, "A finger-ring UWB monopole antenna for BAN and PAN," in Proc. IEEE Antennas Propag. Soc. Int. Symp., Jul. 2010, DOI: 10.1109/APS.2010.5561689.

[10] K. Y. Yazdandoost and K. Hamaguchi, "Very small UWB antenna for WBAN applications," in Proc. Int. Symp. Med. Inf. Commun. Technol., Mar. 2011, pp. 70-73.

[11] A. Fort, C. Desset, J. Ryckaert, P. D. Doncker, L. V. Biesen, and P. Wambacq, "Characterization of the ultra wideband body area propagation channel," in Proc. Int. Conf. Ultrawideband, Zurich, Switzerland, 2005, DOI: 10.1109/ICU.2005.1569950.

[12] A. Fort, J. Ryckaert, C. Desset, P. D. Doncker, P. Wambacq, and L. V. Biesen, "Ultra-wideband channel model for communication around the human body," IEEE J. Sel. Areas Commun., vol. 24, no. 4, pp. 927-933, Apr. 2006

[13] T. S. P. See, A. Alomainy, Y. Hao, and Z. N. Chen, "On-body characterisation of a compact planar UWB antenna," in Proc. Eur. Conf. Antennas Propag., 2006, DOI: 10.1109/EUCAP.2006.4584863.

[14] A. Alomainy, Y. Hao, C. G. Parini, and P. S. Hall, "Comparison between two different antennas for UWB on-body propagation measurements," IEEE Antennas Wireless Propag. Lett., vol. 4, pp. 31-34, 2005.

[15] A. Alomainy, A. Sani, A. Rahman, J. G. Santas, and Y. Hao, "Transient characteristics of wearable antennas and radio propagation channels for ultrawideband body-centric wireless communications," IEEE Trans. Antennas Propag., vol. 57, no. 4, pt. 1, pp. 875-884, Apr. 2009.

[16] T. Tuovinen, K. Y. Yazdandoost, and J. Iinatti, "Monopole ultra wideband antenna for on-body communication in wireless body area network," in Proc. Antennas Propag. Conf., Loughborough, 2011, DOI: 10.1109/LAPC.2011.6114060.

[17] M. Klemm, I. Z. Kovcs, G. F. Pedersen, and G. Tröster, "Novel smallsize directional antenna for UWB WBAN/WPAN applications," IEEE Trans. Antennas Propag., vol. 53, no. 12, pp. 3884-3896, Dec. 2005.

[18] N. Chahat, M. Zhadobov, R. Sauleau, and K. Ito, "A compact UWB antenna for on-body applications," IEEE Trans. Antennas Propag., vol. 59, no. 4, pp. 1123-1131, Apr. 2011.

[19] J.-Y. Sze and K.-L. Wong, "Bandwidth enhancement of a microstripline-fed printed wide-slot antenna," IEEE Trans. Antennas Propag., vol. 49, no. 7, pp. 1020-1024, Jul. 2001.

[20] X. Qing, M. Y. W. Chia, and X. Wu, "Wide-slot antenna for UWB applications," in Proc. IEEE Antennas Propag. Soc. Int. Symp., 2003, pp. 834-837.

[21] R. Chair, A. A. Kishk, and K. F. Lee, "Ultrawide-band coplanar waveguide-fed rectangular slot antenna," IEEE Antennas Wireless Propag. Lett., vol. 3, pp. 227-229, 2004.

[22] R. Chair, A. A. Kishk, K. F. Lee, and C. E. Smith, "Unidirectional wideband slot aperture antennas," in Proc. IEEE Antennas Propag. Soc. Int. Symp., 2004, vol. 2, pp. 1875-1878.

[23] G. Sorbello, M. Pavone, and L. Russello, "Numerical and experimental study of a rectangular slot antenna for UWB communications," $\mathrm{Mi}$ crow. Opt. Technol. Lett., vol. 46, no. 4, pp. 315-319, Aug. 2005, DOI: 10.1002/mop. 20975.

[24] P. S. Hall, H. Yang, Y. I. Nechayev, A. Alomainy, C. C. Constantinou, C. Parini, M. R. Kamarudin, T. Z. Salim, D. T. M. Hee, R. Dubrovka, A. S. Owadally, S. Wei, A. Serra, P. Nepa, M. Gallo, and M. Bozzetti, "Antennas and propagation for on-body communication systems," IEEE Antennas Propag. Mag., vol. 49, no. 3, pp. 41-58, Jun. 2007.

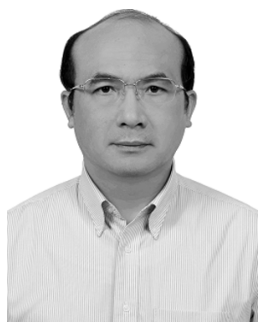

Wen-Tron Shay (M'97) received the B.S. degree in electrophysics from the National Chiao-Tung University, Hsinchu, Taiwan, in 1987, where he is currently working toward the Ph.D. in communication engineering.

Since 1989, he has been with the Electrical \& Electromagnetic Measurement Laboratory, Center for Measurement Standards, Industrial Technology Research Institute, Hsinchu, Taiwan. His curren research interests include electromagnetic field metrology, antennas for body area networks, and 
techniques for measuring and monitoring electromagnetic (EM) radiations from modern wireless communication systems.

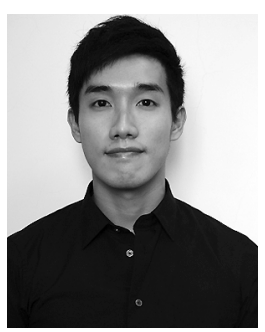

Shiou-Chiau Jan was born in Taipei, Taiwan, in 1986. He received the B.S. degree in electrical engineering from the National Central University, Taoyuan, Taiwan, in 2010 and the M.S. degree in communication engineering from the National Chiao-Tung University, Hsinchu, Taiwan, in 2012.

His current research interests include antennas, indoor on-body radio propagation, and radio-frequency $(\mathrm{RF}) /$ microwave passive circuit components.

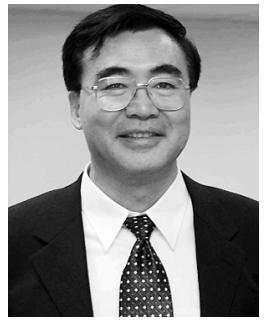

Jenn-Hwan Tarng (S'85-M'86-SM'06) received the Ph.D. degree in electrical engineering from Pennsylvania State University, University Park, PA, USA, in 1989 .

After obtaining the Ph.D. degree, he joined the faculty of the National Chiao-Tung University (NCTU), Hsinchu, Taiwan, where he now holds a position of a Professor in the Department of Electrical Engineering. During 2003-2005, he was the Chairman of the Communication Engineering Department and Director of the Advanced Radio Technology and Systems (ARTS) Center, NCTU, and then he was invited (on leave) as Chair Professor and Dean of Engineering College, Chung-Hua University, Hsinchu, Taiwan, from 2005 to 2007. Since October 2007, he was on leave to the Industrial Technology Research Institute (ITRI) to be the General Director of the Identification and Security Technology Center (ISTC) His professional interests are in medical body area network, radio-frequency identification (RFID)/Internet of Things (IoT), radio channel modeling and measurement, and antenna and radio-frequency integrated circuit (RFIC) design. He has published more than 50 refereed journal papers, 60 conference papers, and three book chapters. He holds one U.S. patent and four U.S. patent applications pending.

Prof. Tarng was the Advanced Program Chair and Technical Program Committee Member and Co-Chair of Antennas and Propagation track for the 2010 Vehicular Technology Conference-Spring (Taipei, Taiwan, May 16-19, 2010). He has also severed as Member of the Technical Planning Committee (TPC) of several IEEE-sponsored technical conferences, including the IEEE International Conference on Networking, Sensing, and Control (ICNSC) and the IEEE International Conference on Systems, Man, and Cybernetics (SMC). He has led and co-led several National Academic Research Programs, such as "Program for Promoting Academic Excellence of Universities-Phases I and II" supported by the Ministry of Education and National Science Council (NSC) of Taiwan. 